\title{
"VALIDACIÓN DE ESTUDIOS INDIVIDUALES DE IMPACTO AMBIENTAL: CASO DEL MEGAPROYECTO DE GAS DE CAMISEA, PERÚ"
}

\author{
Carlos I. Palomares Palomares ${ }^{1}$, Jorge A. Alarcón Novoa ${ }^{2}$
}

Fecha de recepción: 04-04-13

Fecha de aceptación: 20-09-13

\section{Resumen}

El objetivo de este estudio es validar un conjunto de matrices de "Leopold", dado que éstas son el insumo fundamental de los denominados Estudios de Impacto Ambiental, en el marco del Megaproyecto Camisea- Perú. Para ello, se ha utilizado un modelo de regresión probabilístico ("Poisson"), que se basa en datos de recuento (COUNT DATA), con la finalidad de determinar la validez y robustez de los EIA llevados a cabo en el marco de Camisea. El propósito final del trabajo es contribuir con un aporte original a la teoría de la economía ambiental, para que los procesos de fiscalización o regulación que realizan las instituciones peruanas (como OSINERGMIN , MINAM , MEM , PRODUCE , entre otros), sean más eficientes al momento de tomar decisiones de política económica. El estudio utiliza, como ejemplo, tres matrices EIA que corresponden a subproyectos del Lote 88 de CAMISEA. Finalmente, se ha logrado comprobar la importancia y validez del método, mediante el modelo econométrico de "Poisson", el cual se podrá generalizar para validar un conjunto

Máster en Economía del Medio Ambiente y de los Recursos Naturales (Universidad de los Andes - Colombia) Ddirector ejecutivo de Imarpe. Consultor económico ambiental MINAM. Dirección postal: Av. La Molina S/N La Molina (Edif. Facultad de Economía y Planificación - UNALM); Teléfono: (0051) 2213958; e-mail: seramolap70@yahoo.es, cpalomares@minam. gob.pe. distinto de metodologías de valoración cualitativa, como las del BID, CONESA, ERM, Walsh, entre otras.

Palabras clave: estudios de impacto ambiental, matrices de Leopold, evaluación de impacto, proyecto Camisea, recursos naturales comunes.

JEL Classification: Q3, Q4, Q5

\section{Abstract}

Main objective of this study is to validate a set of "Leopold" matrices, given that these are basic elements of so-called Environmental Impact Studies (EIS), under the megaproject "Camisea"- Peru. For this study, it has been used a probabilistic regression model ("Poisson"), which is based on use of "count data", in order to determine the validity and robustness of the EIS, under "Camisea" Project. The ultimate purpose of the paper is to provide an original contribution to the theory of Environmental Economics, for enforcement procedu-

2 Ph.D. en Economía Aplicada (Mississippi State University - USA). Profesor principal e investigador de la Universidad Nacional Agraria La Molina (UNALM - Perú). Dirección postal: Av. La Universidad s/n La Molina Perú. Teléfono: (511) 365-9197; e-mail: jalarcon@lamolina.edu.pe 
res and regulation by Peruvian institutions (as OSINERGMIN MINAM, MEM, among others), be more efficient at when making policy decisions. The study uses, as example, three EIS matrices corresponding to sub-projects Block 88 of Camisea. Finally, it has been proved the validity and importance of the method, using the "Poisson" econometric model, which may be generalized to validate a different set of qualitative valuation methodologies, such as the IDB, CONESA, ERM, Walsh, among others.

Keywords: environmental impact studies, Leopold matrix, impact assessment, Camisea project, common natural resources.

\section{Clasificación JEL: Q3, Q4, Q5}

\section{INTRODUCCIÓN}

En el Perú, como en otros países biodiversos de la región, la medición de impactos de Proyectos de Desarrollo que impliquen alteración en calidad y cantidad de los Recursos Naturales Comunes $(\mathrm{RNC})^{3}$, es una tarea de gran importancia. Lo es también, en el caso estratégico del sector hidrocarburos, el megaproyecto de gas de Camisea en Perú4. En adelante se usa solo la denominación CAMISEA en referencia a este megaproyecto.

\footnotetext{
3 Elinor Ostrom, “El Gobierno de los Comunes", 2000.

4 La explotación de los yacimientos del gas de Camisea, en el Departamento de Cusco- Perú, se inicia en agosto de 2004 veinte años después de su descubrimiento. CAMISEA, es uno de los más importantes Proyectos hidrocarburíficos de América, se encuentra enclavado en el mismo corazón del Departamento del Cusco (en el bajo Urubamba). Los dos lotes más importantes del Mega-proyecto son los lotes 88 y 56, con reservas recuperables de hasta 13.8 trillones de pies cúbicos (TPC). La normatividad permite que el Lote 88 sea enteramente destinado al consumo interno; mientras que el Lote 56 para exportación, a dicho Lote se le suma las nuevas reservas del Lote 57, que explotará REPSOL.
}

Teóricamente, cuando una investigación se refiere a un recurso como el gas natural, es necesario entender las implicancias que tienen las actividades antrópicas en sus diferentes fases, como es el caso de la explotación, y sus impactos en el medio ambiente (biótico y abiótico) y en el medio social, lo cual implica el uso de múltiples criterios para su evaluación. El gas, en esencia, tiene la característica de un bien libre y común en su estado natural, que luego, por entrar en fase de exploración y explotación, se convierte en bien público, lo que causa evidentes externalidades, así como la necesidad de su evaluación. Tal evaluación se efectúa a través de los respectivos Estudios de Impacto Ambiental (EIA), que son las declaraciones juradas de las operadoras que concesionan los lotes y que derivan en metodologías multidisciplinarias aplicadas por tales operadoras, y supervisadas por organismos como el BID, Ministerios de Estado y también Organismos Reguladores, como el Organismo Supervisor de la Inversión en Energía y Minería (OSINERGMIN) y el Organismo de Evaluación y Fiscalización Ambiental (OEFA), en el caso del Perú.

Los diferentes Recursos Naturales Comunes que son parte del transecto por donde se desarrolla el proyecto CAMISEA, representan entes susceptibles de ser impactados, siendo tales impactos medibles con evaluaciones básicamente cualitativas $^{5}$, que según la normatividad peruana, es declarada en todo EIA. Este tipo de evaluación, se efectúa a través de la cualificación de los efectos potenciales de las actividades operativas declaradas por las operadoras durante el desarrollo

\footnotetext{
5 Una evaluación cualitativa se refiere a la valoración numérica subjetiva y categórica de determinado efecto; es decir bueno (1), regular (2), malo (3), por ejemplo. Y que son evaluados según el universo de impactos potenciales en los diversos proyectos que se encuentran inmersos dentro del Megaproyecto Camisea.
} 
del Megaproyecto, en los factores del medio físico (agua, aire y suelo), del medio biológico (flora y fauna) y del medio social (población, salud, identidad, cultura, etc.). Las actividades corresponden a la construcción de ductos, plantas de acopio, pozos, estaciones de bombeo, plantas de fraccionamiento y licuefacción, entre otros. Sin embargo, la evaluación cuantitativa, que es tal vez la más importante (porque debe derivar en una valoración de impactos) es poco usada, a menudo es confusa y ambigua, pues difícilmente refleja el valor más justo a ser remediado, mitigado y/o compensado.

Como en la mayoría de EIA realizados en el Perú, en el caso de CAMISEA sólo se han realizado evaluaciones cualitativas, relacionadas con los diagnósticos de Línea Base que se levantan como parte del trabajo de campo multidisciplinario que realizan las empresas consultoras (por encargo de las "Operadoras"), cuya experiencia se fundamenta en la estructuración de documentos ambientales como los EIA y que tiene connotaciones económicas en el país, debido a que el gas es un buen complemento de la matriz energética del país, que hoy es dependiente del Petróleo. En el caso específico de CAMISEA, la diversidad de sub-proyectos y de empresas consultoras involucradas, hace evidente y compleja la medición de impactos, por lo que una mala decisión podría desencadenar una sub-valoración de los impactos ${ }^{6}$, lo que quita finalmente

\footnotetext{
6 Para el caso de selva, por ejemplo, PLUSPETROL designó a la Empresa Environmental Resources Management (ERM) como la consultora encargada de preparar los EIA de las locaciones en yacimiento, ducto y planta de acopio en "Malvinas". Lo mismo se observa para el caso del ducto donde TGP encargó esta labor a la empresa "WALSH" para el caso del transecto en ceja de selva, sierra y costa. Así mismo, en el caso de costa, los EIA de la planta de fraccionamiento, de muelle y componente fueron encargados a ERM y en el caso de la Planta de Licuefacción en Pampa Melchorita, Perú LNG encargó a Golder Asocciates, la elaboración de tan importante documento ambiental.
}

la sostenibilidad del megaproyecto, y da lugar a potenciales reclamos de compensaciones no bien definidas en los EIA.

Dado que CAMISEA tiene un horizonte intertemporal de 40 años, y a la fecha ya han transcurrido más de ocho años en la fase operativa; es necesario alertar por los efectos generados -o que puedan generarse- debido a la presencia de las actividades hidrocarburíferas que alteren la condiciones de los RNC; lo que en interacción con el hombre, podría causar impactos importantes, como es el caso, por ejemplo, de la deforestación de bosques primarios o secundarios y la intrusión en Reservas Comunales como la de "Nahua Nanti Kugapakori". En tal contexto, son pertinentes las siguientes preguntas: ¿son evaluados adecuadamente los impactos por deforestación?, ¿existe alguna manera de realizar mediciones adecuadas de impacto en los RNC?, ¿serán realistas los EIA de CAMISEA?

En este estudio se hace una propuesta metodológica para validar la certeza y consistencia de estudios cualitativos de impacto, basados en el uso de "Matrices Leopold"7. La aplicación y demostración de la pertinencia del método se ha logrado con un conjunto de matrices de los EIA existentes en el caso del Lote 88 del Proyecto CAMISEA; estas ma-

7 En general una matriz de Leopold es un método de evaluación de impacto ambiental que se utiliza para identificar el impacto inicial de un proyecto en un entorno natural. El sistema consiste en una matriz con columnas representando varias actividades que ejerce un proyecto (por ejemplo, extracción de tierras, incremento del tráfico, ruido, polvo, etc), y en las filas se representan varios factores ambientales que son considerados (aire, agua, geología, etc.). Las intersecciones entre ambas se numeran con dos valores, uno indica la magnitud (de -10 a +10 , por ejemplo) y el segundo la importancia (de 1 a 10 , por ejemplo) del impacto de la actividad respecto a cada factor ambiental. 
trices han sido el insumo fundamental de la evaluación de los impactos descritos en los EIA y sirven para cuantificar los impactos económicos sobre los medios afectados o FAS ${ }^{8}$. Para ello, se ha utilizado un modelo de regresión probabilístico (Modelo de "Poisson"), que utiliza datos de "recuento"; con esto, se ofrece al mundo científico y decisor un aporte metodológico original para que los procesos de fiscalización o regulación que realizan las entidades nacionales del Perú (como OSINERGMIN, Ministerios del Ambiente, Energía y Minas, PRODUCE ${ }^{9}$, entre otros), sean más eficientes al momento de tomar decisiones de política pública y económica que involucren, por ejemplo, una multa por infracción ante la presencia de impactos no previstos, o ante el incumplimiento de compromisos declarados en los respectivos documentos ambientales. Más que la pretensión de evaluar la mayor cantidad posible de sub-proyectos de CAMISEA (que es propósito de una tesis de doctorado UNAM-UNALM ${ }^{10}$ ), en este caso el propósito es presentar, en forma objetiva y didáctica, una metodología que potencialmente puede ser utilizada para evaluaciones en proyectos de desarrollo similares en los sectores energéticos y mineros del Perú. Eventualmente el método podría ser adecuado y utilizado en las circunstancias propias de otros países de la región.

Este artículo contiene cinco secciones, incluyendo la introducción. La segunda sección

8 Factor Ambiental y/o Social, tal como el agua superficial, agua subterránea, aire, suelo, flora, fauna, etc.

9 Ministerio de la Producción (PRODUCE)

10 Palomares, Carlos (tesis no publicada). "Medición de Impactos en los Recursos Naturales Comunes del Proyecto Camisea-Perú, a Través de la Evaluación de Estudios Individuales de Impacto Ambiental, Utilizando Datos Panel"(borrador final de TESIS). presenta una revisión concisa de literatura, sobre la que se sustenta el tema de la investigación. La metodología es presentada en la tercera sección; luego, en la cuarta sección se presentan los resultados obtenidos a partir del modelo de regresión utilizado, así como la discusión y análisis de los resultados. La sección cinco presenta las conclusiones extraídas del análisis de resultados.

\section{MARCO TEÓRICO Y METODOLÓGICO}

\subsection{En relación a la medición cualitativa de impactos}

Como en la mayoría de Proyectos con hidrocarburos en el Perú, en el caso del Proyecto CAMISEA los Estudios de Evaluación de Impacto Ambiental se han estructurado y sistematizado en matrices de Leopold ${ }^{11}$, elaboradas en base a distintos métodos de cualificación, y por equipos multidisciplinarios de consultoras privadas (como Environmental Resources Managment, WALSH, entre otras). Para el propósito de este estudio, los resultados sistematizados en las matrices Leopold sirven de insumo para evaluar la consistencia de la información cualitativa existente, incluyendo la comparación con información proveniente de metodologías más estandarizadas, como la del Banco Interamericano de Desarrollo (BID), que ha tenido aplicaciones en proyectos hidrocarburíficos ${ }^{12}$.

11 En el Perú, una de las matrices más usadas es la de Leopold, que recoge, por ejemplo, acciones que pueden ser relacionadas con factores ambientales en un número variable, que depende del tipo de proyecto.

12 Otra metodología estandarizada, que también ha sido ampliamente utilizada para elaboración de EIA (sobre todo en el campo del sector minero) es el propuesto por Víctor Conesa Fernández (2010). 
El impacto o "Índice de Incidencia" utilizado en los EIA, puede definirse como el ratio mediante el cual se mide cualitativamente el impacto ambiental, en función, tanto del grado de incidencia o intensidad de la alteración producida, como de la caracterización del efecto (que responde a su vez a una serie de atributos de tipo cualitativo, tales como extensión, tipo de efecto, plazo de manifestación, persistencia, reversibilidad, recuperación, sinergia, acumulación, periodicidad, entre otros dependiendo del método). A pesar que los diferentes métodos utilizan criterios distintos para elaborar las matrices de Leopold, todas las metodologías coinciden en un análisis y enfoque sistémico que involucra la evaluación de las sinergias en los tres medios del ecosistema como son: el medio físico, biológico y social que se establece en el contexto de un universo de impacto, por lo cual, se puede estandarizar la evaluación propuesta para cualquier tipo de metodología utilizada en su elaboración.

Siendo el propósito de este estudio validar la consistencia y magnitud de impactos de los EIA, mediante el uso de una metodología implementada con instrumental econométrico, la estructura de la información existente ("recuento de datos") permite realizar tal validación objetiva y consistente de los impactos generados en los RNC, por presencia específica de algunos subproyectos de CAMISEA. Esta misma propuesta, será posible aplicar a los distintos subproyectos en los cuales se han utilizado metodologías reconocidas como de mayor fortaleza teórica, como es el caso de CONESA o la del $\mathrm{BID}^{13}$.

\subsubsection{Metodología de Evaluación de Impactos del Banco Interamericano de Desarrollo (BID)}

Para efectos ilustrativos y comparativos de métodos cualitativos en el desarrollo de proyectos que involucren RNC, se presenta la metodología propuesta y utilizada por el BID. En este caso, la evaluación de los impactos es definida en base a múltiples criterios que asignan un valor numérico discreto teniendo en cuenta el grado de ocurrencia, severidad y las posibles medidas de control, según los valores que son presentados en la Tabla 1, a continuación.

\footnotetext{
13 En CAMISEA como en otros proyectos peruanos con hidrocarburos, las metodologías utilizadas por la mayoría de Consultoras se caracterizan por que van variando acorde al tiempo y circunstancias, siendo unas más exigentes y rigurosas que otras. Los métodos de CONESA y del BID han sido muchos más sostenibles, complejas y de mayor rigurosidad que otros métodos utilizados en la región.
} 


\section{Tabla 1. Criterios de Evaluación de Impactos Ambientales y Sociales según el BID}

\begin{tabular}{|c|c|c|c|}
\hline \multicolumn{3}{|c|}{ CRITERIOS } & \multirow{2}{*}{$\begin{array}{c}\text { VALOR } \\
0\end{array}$} \\
\hline \multirow{24}{*}{ Ocurrencia } & \multirow{5}{*}{ Probabilidad de ocurrencia (Pr) } & No ocurrirá & \\
\hline & & Baja probabilidad de ocurrencia & 2 \\
\hline & & Mediana probabilidad de ocurrencia & 5 \\
\hline & & Alta probabilidad de ocurrencia & 8 \\
\hline & & Certeza de ocurrencia & 10 \\
\hline & \multirow{5}{*}{ Duración del Impacto (Du) } & Instantáneo & 0 \\
\hline & & Corto plazo (< 1 año) & 2 \\
\hline & & Mediano Plazo (< 5 años) & 5 \\
\hline & & Largo Plazo (< 10 años) & 8 \\
\hline & & Permanente/Irreversible & 10 \\
\hline & \multirow{7}{*}{ Extensión del Impacto (Ex) } & $10 \mathrm{~m}$ de radio & 0 \\
\hline & & $100 \mathrm{~m}$ de radio & 1 \\
\hline & & $1 \mathrm{~km}$ de radio & 2 \\
\hline & & En un sector de la ciudad & 3 \\
\hline & & En toda la ciudad & 5 \\
\hline & & Regional & 8 \\
\hline & & Nacional/Internacional & 10 \\
\hline & \multirow{7}{*}{ Población Impactada (Po) } & 0 habitantes & 0 \\
\hline & & $<50$ habitantes & 1 \\
\hline & & $<200$ habitantes & 2 \\
\hline & & $<50000$ habitantes & 3 \\
\hline & & $<350000$ habitantes & 5 \\
\hline & & $<1000000$ habitantes & 8 \\
\hline & & $>1000000$ habitantes & 10 \\
\hline \multirow{16}{*}{ Severidad } & \multirow{4}{*}{ Impacto a la salud Humana (Ih) } & Mejoramiento sustancial/Potencialmente fatal & $10 /-10$ \\
\hline & & Mejoramiento mayor/Deterioro mayor & $7 /-7$ \\
\hline & & Mejoramiento menor/Deterioro menor & $2 /-2$ \\
\hline & & Sin impacto & 0 \\
\hline & \multirow{4}{*}{ Impacto al ecosistema (le) } & $\begin{array}{l}\text { Mejoramiento sustancial/Deterioro mayor } \\
\text { Ecosistemas sensibles }\end{array}$ & $10 /-10$ \\
\hline & & Mejoramiento mayor/Deterioro mayor & $7 /-7$ \\
\hline & & Mejoramiento mínimo/Deterioro menor & $2 /-2$ \\
\hline & & Sin impacto & 0 \\
\hline & \multirow{4}{*}{ Impacto Sociocultural (Is) } & Mejoramiento sustancial/Pérdida total de recursos & $10 /-10$ \\
\hline & & Mejoramiento mayor/Deterioro mayor de recursos & $7 /-7$ \\
\hline & & Mejoramiento menor/Deterioro menor de recursos & $2 /-2$ \\
\hline & & Sin impacto & 0 \\
\hline & \multirow{4}{*}{ Impacto Económico (Ic) } & Mejoramiento sustancial/Deterioro catastrófico & $10 /-10$ \\
\hline & & Mejoramiento mayor/Deterioro mayor de recursos & $7 /-7$ \\
\hline & & Mejoramiento menor/Deterioro menor de recursos & $2 /-2$ \\
\hline & & Sin impacto & 0 \\
\hline
\end{tabular}




\begin{tabular}{|c|c|c|c|}
\hline \multicolumn{3}{|c|}{ CRITERIOS } & VALOR \\
\hline \multirow{18}{*}{$\begin{array}{l}\text { Medidas de } \\
\text { Control }\end{array}$} & \multirow{5}{*}{ Medidas de prevención (Mp) } & Es posible prevención completa & $0 / 0$ \\
\hline & & Es posible prevención extensiva & $0 / 2$ \\
\hline & & Es posible prevención parcial & $0 / 5$ \\
\hline & & Es posible prevención temporal & $0 / 8$ \\
\hline & & Medidas ineficaces o no disponibles & $0 / 10$ \\
\hline & \multirow{5}{*}{ Medidas de mitigación (Mm) } & Es posible mitigación completa & 0 \\
\hline & & Es posible mitigación extensiva & $0 / 2$ \\
\hline & & Es posible mitigación parcial & $0 / 5$ \\
\hline & & Es posible mitigación temporal & $0 / 8$ \\
\hline & & Mitigación ineficaz o no disponible & $0 / 10$ \\
\hline & \multirow{4}{*}{ Medidas de mantenimiento (Mt) } & No se requiere mantenimiento & $10 / 0$ \\
\hline & & Mínimo mantenimiento en ejecución & $8 / 5$ \\
\hline & & Algún mantenimiento en ejecución & $5 / 8$ \\
\hline & & Mantenimiento extensivo requerido en ejecución & $0 / 10$ \\
\hline & \multirow{4}{*}{ Medidas de monitoreo (Mn) } & No se requiere monitoreo & $10 / 0$ \\
\hline & & Mínimo monitoreo en ejecución & $8 / 5$ \\
\hline & & Algún monitoreo en ejecución & $5 / 8$ \\
\hline & & Monitoreo extensivo requerido en ejecución & $0 / 10$ \\
\hline
\end{tabular}

Fuente: Banco Interamericano de Desarrollo. Elaboración Propia.

A partir de estos valores establecidos, se estiman los potenciales impactos de las actividades, en los factores ambientales y sociales que se colocan en la matriz de impacto o Leopold, según la siguiente ecuación:

$$
\text { Valoración }=\frac{(P r+D u+E x+P o) x(I h+I e+I s+I c) x(M p+M m+M t+M n)}{1000}
$$

Tal cualificación de impactos ambientales y sociales, permite categorizar las perturbaciones ocasionadas por las actividades del pro- yecto, en cada uno de los factores a evaluar, según los rangos de importancia mostrados en la Tabla 2.

\section{Tabla 2. Rangos de Importancia según Método de Evaluación BID, de impactos}

\begin{tabular}{l|c|c|}
\hline \multirow{2}{*}{ Categoría de Importancia } & \multicolumn{2}{|c}{ Resultado del Impacto } \\
\cline { 2 - 3 } & Negativo & Positivo \\
\hline Extrema & $<=-15,0$ & $>=+15,0$ \\
Alta & $<=-5,0$ & $>=+5,0$ \\
Media & $<=-1,0$ & $>=+1,0$ \\
Baja & $>-1,0$ & 0 a 1,0
\end{tabular}


Una vez cuantificados los impactos ambientales y sociales, según los rangos de importancia establecidos, se estructura la matriz de impactos con los resultados para identificar los parámetros estimados de impacto en los componentes ambientales y sociales, por la presencia de las actividades de un proyecto.

\subsubsection{Metodologías más usadas en los Sub-proyectos de CAMISEA}

Como fue mencionado antes, en el Perú las empresas operadoras de los Proyectos de Desarrollo en Hidrocarburos, han requerido los servicios de consultoras ambientales, nacionales e internacionales, para cumplir con realizar sus diferentes Estudios de Impacto Ambiental, que se evalúan sectorialmente, según lo estipula la norma. En el caso de CAMISEA, en cada uno de los Sub Proyectos que son parte de todo su espectro y en sus transectos regionales de Selva, Sierra y Costa; por encargo contractual, las consultoras ambientales utilizan metodologías que se caracterizan porque van variando acorde al tiempo y circunstancias, siendo unas más exigentes y rigurosas que otras. Las empresas consultoras utilizan métodos cualitativos sumamente simples en algunos casos y más complejos en otros (los métodos de CONESA y del BID parecen haber sido mucho más sostenibles). Entre las principales empresas ambientales requeridas para la elaboración de EIA en CAMISEA, se encuentran: Environmental Resources Managenment (ERM), Walsh Perú y Golder Associates, entre otras.
Si bien es cierto, cada una de estas instituciones privadas han realizado muchos EIA, tales estudios presentan diferencias en la forma de estructurar las matrices de impacto, ya que se basan en metodologías propias derivadas del método original de Leopold, que han ido modificando y mejorando con el tiempo, con el objeto de ser cada vez más consistentes. Con esta investigación, se han evaluado algunas de las propuestas de CAMISEA (Lote 88), que pueden ser comparadas con otras metodologías como la del Banco Interamericano de Desarrollo, que se ciñe a los procesos de cualificación moderna o contemporánea en el medio latinoamericano.

\subsection{CON RELACION A LA ECONOMETRIA PARA EVALUAR Y CUANTIFICAR IMPACTOS}

\subsubsection{Método Econométrico para validar las Matrices de Impactos}

El modelo de Poisson, presenta una alternativa metodológica probabilística, con variable dependiente o variable objetivo discreta (se llama "de cuenta" pues tiene pocos números, enteros, positivos). Se basa en una distribución de probabilidades que ha sido probada útil cuando la variable dependiente representa "datos de cuenta" ó "recuento de datos"(Count Data en inglés); es decir, en el caso que la variable discreta toma solo un número finito de valores representados por números enteros (Guja- 
rati, 2009) ${ }^{14}$.El modelo de Poisson, ha sido mayormente aplicado para estimar probabilidades asociadas a estudios relacionados con la salud humana ${ }^{15}$ (Cameron et al, 2013; Pohlmeier y Ulrich, 1995; Gerdtham, 1997; Urbanos, 2000 y Álvarez, 2001).

En Economía Ambiental, este modelo ha sido frecuentemente utilizado como instrumento del método de "costo de viaje" para calcular el valor económico de los servicios que brindan bienes que usualmente no son transados en el mercado, como los lugares de recreación, parques, playas, lagos y otras áreas públicas (Riera et.al., 2006). En esta oportunidad; el modelo de Poisson es utilizado, en forma original y atractiva, para validar EIA, es decir la "idoneidad" de los impactos ocasionados por las actividades del Proyecto CAMISEA.

El modelo de regresión no es utilizado en su forma clásica, en el que se recoge información que sirve para alimentar y construir una variable dependiente y un conjunto de variables regresoras, con el previo establecimiento de una teoría que sustenta una relación de causalidad. Los datos en este caso provienen de valoraciones cualitativas expresadas en las matrices de Leopold; las variables regresoras se construyen a partir de la información provista en las matrices (se estructuran en función al conjunto de actividades que realicen las operadoras afectando al medio eco-sisté-

\footnotetext{
14 La variable discreta toma un número finito de valores y representa fenómenos frecuentes (como el número de patentes otorgadas a una empresa en un año, número de visitas a un dentista en una año, número de visitas a un supermercado en una semana) o también ocurrencias poco frecuentes, como ganar la lotería o tener un ataque al corazón en un trimestre, etc.

15 Como es el caso de número de consultas médicas, al número de ingresos hospitalarios, días de estancia y número de medicamentos consumidos, entre otros).
}

mico en su conjunto), mientras que la variable dependiente es una variable de "cuenta", que expresa el número de veces que se producen impactos sobre un determinado factor ambiental y/o social FAS. No hay una teoría económica detrás, pues es un caso típico y sui-géneris del uso de un modelo de Variable Dependiente discreta, que es utilizado para comprobar si existe una relación significativa entre regresoras y variable dependiente. De existir tal relación se valida la certeza y precisión de la matriz de Leopold (y por tanto, del estudio de impacto).

Más específicamente, el uso de un modelo de regresión de Poisson permite identificar las pautas convencionales para evaluar y validar los EIA formulados a través de las declaraciones de Línea Base, que son sistematizados en las matrices de impacto (o matrices de Leopold), en la cual se relacionan los FAS en función al conjunto de actividades que impactan potencialmente a los tres medios eco-sistémicos (físico, biológico y social). El modelo permite la identificación del número de impactos que llegan a producirse en los diferentes bienes y servicios eco-sistémicos o recursos naturales comunes que se encuentren inmersos en el ámbito del proyecto. Tales impactos, se identifican a través de los factores ambientales y sociales descritos en las matrices de Leopold y elaborados por múltiples criterios propuestos por el equipo multidisciplinario. El modelo requiere que la distribución condicional para la variable de respuesta sea correctamente especificada, así como el parámetro relacionado con su valor esperado. 
Para la regresión Poisson se asume que la distribución condicional de $\left(y_{i} / x_{i}\right)$ se distribuye como una variable aleatoria Poisson con función de densidad:

$$
f\left(y_{i} / x_{i}\right)=\frac{e^{-\lambda_{i} \lambda_{i}{ }^{y}}}{y !}, \forall y=0,1,2, \ldots(1)
$$

Donde $y$ ! es el factorial, expresado por $y !=y^{*}(y-1) *(y-2) * \ldots * 2 * 1$

$\lambda$ es el número promedio de impactos que se generan en un periodo de tiempo ${ }^{16}$. El número de impactos se caracteriza por ser finito y responderá a la estructura potencial de los impactos mediante una formalización matemática que responden a los criterios de afectación específica o combinada de las actividades sobre los medios físico, biológico y social, y que permitan inferir decisiones de política en la sostenibilidad del proyecto ${ }^{17}$. Si el valor de $y_{i}$ fuera alto, las probabilidades asignadas por este modelo son tan bajas como se desee, es decir tenderían a cero (Greene, 2004).

A partir de la ecuación (1) es posible establecer el parámetro para la media condicional:

$$
E\left[y_{i} /\left(x_{i}\right)\right]=\lambda_{i}=\exp \left(x_{i}^{\prime} \beta\right)
$$

Dada la especificación de la distribución condicional de la variable respuesta, así como de la media condicional, bajo el supuesto de que se tienen observaciones independientes, entonces se pueden encontrar los estimadores de los parámetros de "causalidad" $(\beta)$, utili-

\footnotetext{
16 El período del EIA, que se diferencia por tipo de proyecto, según sea pequeño, mediano o grande.

17 G. Munda (2008), "Social Multi-Criteria evaluation for a sustainable economy".
}

zando una función de Máxima Verosimilitud (expresada en Logaritmos y cuya robustez se analiza por la distribución Chi cuadrada ): $\square_{i}^{2}$

$L(\beta)=\sum_{i=1}^{n}\left(y_{i} x_{i}^{\prime}-\exp \left(x_{i}^{\prime} \beta\right)-\log \left(y_{i}^{\prime}\right)\right)$

Es decir, que los estimadores $(\beta)$ y sus respectivos errores estándar son obtenidos a partir de la maximización de la función de verosimilitud (3). Tales estimadores tienen las propiedades de consistencia y eficiencia asintótica (Wooldridge, 2009).

En el caso de una función Poisson, es posible demostrar que la media y varianza, son iguales: $\operatorname{Var}\left(y_{i}\right)=\lambda=E\left(y_{i}\right)$, lo cual conlleva la necesidad de corregir los errores estándar asociados a los coeficientes estimados (errores estándar "robustos").

Entonces, la forma operativa del modelo de regresión puede también expresarse como:

$\lambda_{i}=E\left(Y_{i}\right)=e^{\beta_{1}+\beta_{2} X_{1 i}+\beta_{3} X_{2 i}+\cdots+\beta_{k-1} X_{k i}}$

En el caso específico de este estudio, las variables $X$ son las variables "explicativas" que afectan el valor promedio de los impactos $\left(\lambda_{i}\right)$, dado que en este caso particular la variable dependiente representa el número de impactos generados por las actividades descritas en la Matriz de Leopold, que ocasionan "externalidades" en un período determinado por la evaluación de lo descrito en la línea base del EIA ${ }^{18}$.Finalmente, los resultados permitirán al evaluador del EIA, tomar decisiones

\footnotetext{
18 El período dependerá de la fase que se esté evaluando, la cual puede clasificarse en: Fase de Exploración, Fase de Construcción, Fase de Operación y Fase de Abandono o Cierre.
} 
sobre la viabilidad del Proyecto, o dar recomendaciones a tener en consideración sobre la mitigación de impactos en ciertos FAS.

\section{METODOLOGÍA}

El estudio parte del hecho que es necesario, para evaluadores de impactos ambientales, y para quienes realizan tales estudios, contar con un instrumento metodológico de validación de lo realizado a través de las matrices de impacto; es decir que les permita, desde la óptica del Estado, asegurarse que la evaluación realizada sea consistente y confiable. Se plantea entonces un procedimiento de validación utilizando el Método de "recuento de datos", en base a una distribución de Poisson, que permite contabilizar el número de ocurrencias por unidad de tiempo. En este caso particular, se contabiliza el número de impactos evaluados durante el período de elaboración del EIA ${ }^{19}$. Un supuesto importante es que el número de impactos medidos o "capturados" en la variable dependiente $(Y)$, tomados a partir de información provista por las Líneas de Base de los diferentes subproyectos, es bastante aproximado a los impactos reales de las actividades. De no ser así, podría caerse en un problema de regresión "espuria"20.

\subsection{Especificación del Modelo}

Como fue expresado en la sección teórica anterior, el modelo de Poisson operativo o funcional puede ser representado por la ecuación exponencial (2) anterior:

$$
\left[y_{i} /\left(x_{i}\right)\right]=\lambda_{i}=\exp \left(x_{i}^{\prime} \beta\right)
$$

\section{Donde:}

$y_{i}=$ número total de veces que el factor " $i$ " (del medio ambiental o social) es impactado por el total de las " $m$ " actividades consideradas en la matriz Leopold ${ }^{21}$.

$X_{i}=$ magnitud del efecto de " $k$ " actividades que afectan a un FAS/ $N^{\circ}$ de veces que el factor " $\boldsymbol{i}$ " es impactado por " $\boldsymbol{k}$ " actividades; es decir:

$$
x_{i}=\frac{\text { Magnitud }(\Sigma) \text { de las valoraciones correspond. al efecto de } " k " \text { Activ. que afectan a un det. factor }}{N^{\circ} \text { total de veces que el factor } " i " \text { es impactado por } " k " \text { actividades que afectan a un det. factor }}
$$

$m=$ número total de actividades que impactan los factores (físicos, biológicos y sociales) en la matriz de Leopold $n=$ número de factores impactados declara-

19 La alternativa ha sido el uso de un modelo de distribución BINOMIAL NEGATIVA, descartada porque limita al modelo hasta que ocurra el impacto considerando como límite el primer éxito (E) de impacto, lo cual no sería apropiado para el objetivo de este estudio.

20 Es importante recordar acá nuevamente, que se necesita suponer que el número de impactos medidos o "capturados" en la variable dependiente, tomados a partir de información provista por los propios EIA, basados en las Líneas de Base de los subproyectos, es bastante aproximado a los impactos reales de las actividades. dos en el EIA

$k=$ parte del total de " $m$ " actividades que afectan a un determinado factor o medio $(k<m)$

$i=1$ a $n$ (corte transversal).

Dado que a través del modelo se validan los

21 Esta es una denominación usada frecuentemente en el contexto de trabajo con series de tiempo, pero también eventualmente utilizada en otros tipos de regresión en los cuales los resultados de la relación entre variables es de dudoso valor, en el sentido que los resultados parecen, a primera vista, buenos, pero una investigación más detenida revela que resultan sospechosos (Gujarati, 2010). 
impactos potenciales de las actividades (o grupo de actividades) de un Proyecto, la variable dependiente o endógena (denominada "FACT") es definida por el conteo de impactos generados por los grupos de dichas actividades descritas en la Matriz de Leopold, y que ocasionan externalidades en los diferentes factores ambientales y sociales. El nivel de conteo de impactos dependerá del conjunto de actividades que se generen en cada uno de los factores ambientales y sociales de los tres medios eco-sistémicos (el medio físico, biológico y social); también dependerá de los posibles impactos combinados que pueda generar entre los medios antes mencionados.

Las $X_{i}$ son las variables explicativas del modelo, se construyen según la relación expresada líneas arriba, representado externalidades ocasionadas (por las actividades agrupadas o simples) en los diferentes factores ambientales y sociales, en un período determinado, por la línea base del EIA ${ }^{22}$. La relación entre $x_{i}$ y la "variable de cuenta"(dependiente, $y_{i}$ ) es establecida mediante la estimación de los parámetros del modelo.

Cumpliendo con el principio de "parsimonia" y a fin de contar con variables exógenas o regresoras consistentes, éstas han sido agrupaa das o asociadas, evitándose así perder grados de libertad en el modelo. Tal agrupación, se ha hecho en base a los impactos declarados para los factores del medio eco-sistémico, ocasionado por el conjunto de actividades de los diferentes subproyectos. En este caso se describe a los grupos del siguiente modo:

22 El período dependerá de la fase que se esté evaluando, la cual puede clasificarse en: Fase de Exploración, Fase de Construcción, Fase de Operación y Fase de Abandono o Cierre.
- GP1: Grupo de actividades que afectan al medio Físico, Biológico y Social

- GP2: Grupo de actividades que afectan al medio Físico y Biológico

- GP3: Grupo de actividades que afectan al medio Biológico y Social

- GP4: Grupo de actividades que afectan al medio Físico y Social

- GP5: Grupo de actividades que afectan al medio Físico

- GP6: Grupo de actividades que afectan al medio Biológico

- GP7: Grupo de actividades que afectan al medio Social

El modelo de regresión Poisson con datos de recuento, en este caso, permite tener una visión de cómo es la estructura propuesta por los evaluadores del EIA, donde el equipo multidisciplinario ha colocado una opinión muy particular respecto a su especialidad (expresada en las matriz de Leopold, que es resultado de lo evaluado -por estos expertos- en lo que respecta al impacto en el medio físico, biológico y social). En tal caso, las hipótesis nulas respecto a los parámetros del modelo 2 son simples y pueden ser consideradas del siguiente modo:

$$
\text { Ho: } \beta_{j}=0 ; \text { Ha: } \beta_{j} \neq 0 \text {. }
$$

La hipótesis nula (Ho) equivale a plantear que el impacto previsto a partir de actividades que afectan a un determinado factor (ambiental, social o físico) no se aproxima a lo realmente evidenciado. Por tanto, la evaluación del grupo disciplinario, en este sub-proyecto, no habría sido el más adecuado. En otras palabras, de no rechazarse la hipótesis nula, es 
recomendable tener cautela con el proceso de monitoreo de las actividades que pertenecen al grupo "regresor" durante el tiempo que dure el proyecto.

\subsection{Indicadores relevantes}

En cuanto a la interpretación de resultados para la validación de matrices, éstos serían:

a) Con respecto a su media, el análisis funcional está representado por:

$$
\lambda_{i}=e^{\beta_{0}+\beta_{1} X_{1 i}+\cdots+\beta_{k} X_{k i}}
$$

b) Para determinar la probabilidad de ocurrencia de los impactos en los factores ambientales, por parte del conjunto de actividades de los diferentes subproyectos de Camisea, puede usarse:

$$
P\left(Y_{i}<K\right)=\sum_{i=0}^{k} \frac{e^{-\lambda_{i}} \lambda_{i}^{y}}{y !}
$$

c) En el caso de los efectos marginales que una variable regresora tiene sobre los valores medios de la variable dependiente, éstos son estimados en función a las derivadas parciales siguientes:

$$
\frac{\partial \lambda_{i}}{\partial X_{j i}}=\beta_{k} \lambda_{i}
$$

Esto explicaría que la tasa de cambio del valor medio de los impactos, respecto a las variables independientes, que representan el conjunto de actividades que afectan los factores, es igual al coeficiente de esa regresora multiplicada por su valor medio.

\subsection{Base de Datos}

El proceso metodológico, en el presente caso, ha incluido los siguientes aspectos:

Revisión de los Estudios de Impacto Ambiental y Social de las Operadoras de Gas Natural en el ámbito del Proyecto CAMISEA

Identificación de los impactos en los RNC o FAS, realizados por los equipos multidisciplinarios que elaboraron los EIA (implica evaluar las matrices de impacto, homogeneizándolas en su evaluación)

Validación de los Estudios de Impactos Ambientales (EIA), mediante el uso de un modelo de POISSON, que utiliza "datos de cuenta" proveídos por las evaluaciones de los sub-proyectos.

En forma específica, en la primera etapa se requirió del acopio de los EIA del proyecto Camisea. Para ello, se tuvo que acceder a información pública de los respectivos documentos ambientales que se encontraban en los organismos competentes (como el MEM y OSINERGMIN). Sólo cuando no hubo información precisa y completa, se solicitó el apoyo de algunas empresas operadoras del rubro. Luego de ello, en la fase 2 , se pasó a identificar los impactos que las actividades del sector hidrocarburos pueden generar en los RNC, del Medio Físico y Biológico, con implicancias en el medio social; a través de la descripción desarrollada por el equipo multidisciplinario de la consultora que elaboró el EIA. 
Después del acopio de los documentos ambientales, se empezó a construir la base de datos respectiva, a fin de poder sistematizar la información primaria para evaluar la validación de las matrices de impacto. Dicha validación de matrices, permite dar mayor consistencia y objetividad a los EIA, ya que a través de las técnicas econométricas utilizadas, se logra evaluar la robustez global y significancia de las variables exógenas que son representadas por las actividades del sector, y que ocasionan externalidades en los factores ambientales y sociales de los respectivos sub-proyectos de Camisea.

En este estudio, se han tomado como ejemplo ilustrativo, de aplicación metodológica, dos sub-proyectos del Lote 88 de CAMISEA. Para lograr tal propósito se ha requerido contar con estudios de impacto ambiental, planes de manejo ambiental, programas de relaciones comunitarias de las áreas involucradas, y documentos ambientales ${ }^{23}$ sistematizados, como antedicho, por instituciones como OSINERGMIN y el MEM. A modo de ejemplo, la matriz de evaluación de impactos (Leopold) de uno de los subproyectos es presentada en el Anexo de la última página.

\section{RESULTADOS}

Uno de los dos lotes más importantes en el yacimiento de Camisea, es el Lote 88; por lo que, es de especial interés entender objetivamente las evaluaciones realizadas en los respectivos documentos ambientales, según el tipo de sub proyecto y la relación entre activi- dades impactantes en los factores ambientales y sociales que son parte de los EIA. Como antedicho, en este caso y con propósito ilustrativo, los resultados son presentados para evaluación de dos sub-proyectos del Lote 88 y un sub-proyecto que utiliza la metodología $\mathrm{BID}^{24}$.Los resultados del modelo de regresión, obtenidos con el software STATA, se sintetizan en la siguiente Tabla 3.

En general en estos tres casos la ecuación evaluada ha sido la siguiente:

$E\left(F A C T_{i}\right)=\beta_{0}+\beta_{1} G P 1+\beta_{1} G P 2+\beta_{3} G P 3+$ $\beta_{4} G P 4+\beta_{5} G P 5+\beta_{6} G P 6+\beta_{7} G P 7+\mu_{i}$

\section{Donde:}

GP1: Grupo de actividades que afectan al medio Físico, Biológico y Social

GP2: Grupo de actividades que afectan al medio Físico y Biológico

GP3: Grupo de actividades que afectan al medio Biológico y Social

GP4: Grupo de actividades que afectan al medio Físico y Social

GP5: Grupo de actividades que afectan solo al medio Físico

GP6: Grupo de actividades que afectan solo al medio Biológico

GP7: Grupo de actividades que afectan solo al medio Social

Los dos subproyectos incorporados en esta evaluación pertenecen al Lote 88 de CAMISEA; son los siguientes:

24 En la tesis no publicada de Carlos Palomares "(2013), se incluyen resultados de aplicación del método en un total de 29 subproyectos de CAMISEA (19 del Lote 56 y 10 del Lote 88 ). 
- Subproyecto de Construcción de la planta de gas y anexos (Matriz "Planta de Gas")

- Subproyecto de Operación de Líneas de Conducción (Matriz "Línea de conducción").

Los resultados son mostrados en la Tabla 3, a continuación.

Tabla 3. Resultados de Evaluación de dos Matrices de Impacto del Lote 88

\section{(Regresión Poisson)}

\begin{tabular}{|c|c|c|c|c|c|c|}
\hline \multirow[b]{2}{*}{ Subproyecto } & \multicolumn{3}{|c|}{ Coeficientes de Regresión } & \multicolumn{3}{|c|}{ Efectos Marginales } \\
\hline & $\begin{array}{l}\text { Matriz "Planta } \\
\text { de Gas" }\end{array}$ & $\begin{array}{c}\text { Matriz } \\
\text { "Línea de } \\
\text { Conducción" }\end{array}$ & $\begin{array}{c}\text { Matriz } \\
\text { BID }\end{array}$ & $\begin{array}{l}\text { Matriz "Planta } \\
\text { de Gas" }\end{array}$ & $\begin{array}{l}\text { Matriz "Línea de } \\
\text { Conducción" }\end{array}$ & $\begin{array}{c}\text { Matriz } \\
\text { BID }\end{array}$ \\
\hline VARIABLES & FACT & FACT & FACT & $\frac{\partial \lambda_{i}}{\partial X_{i i}}$ & $\frac{\partial \lambda_{i}}{\partial X_{i i}}$ & $\frac{\partial \lambda_{i}}{\partial X_{i i}}$ \\
\hline GP1 & $\begin{array}{l}-0.884^{1 /} \\
(0.188)\end{array}$ & $\begin{array}{l}-0.808^{2 /} \\
(0.316)\end{array}$ & $\begin{array}{l}-0.572^{1 /} \\
(0.150)\end{array}$ & -7.13 & -2.65 & -2.81 \\
\hline GP2 & $\begin{array}{l}-0.0605 \\
(0.111)^{\mathrm{NS}}\end{array}$ & & $\begin{array}{l}-0.204^{*} \\
(0.107)\end{array}$ & -0.49 & & -1.00 \\
\hline GP3 & $\begin{array}{l}-0.501^{1 /} \\
(0.155)\end{array}$ & $\begin{array}{l}-0.321^{1 /} \\
(0.117)\end{array}$ & $\begin{array}{l}-0.175^{3 /} \\
(0.098)\end{array}$ & -4.04 & -1.05 & -0.86 \\
\hline GP4 & & $\begin{array}{l}-0.332^{2 /} \\
(0.150)\end{array}$ & $\begin{array}{l}-0.302^{2 /} \\
(0.132)\end{array}$ & & -1.09 & -1.49 \\
\hline GP5 & $\begin{array}{c}-0.395 \\
(0.498)^{\mathrm{NS}}\end{array}$ & $\begin{array}{l}-0.661 \\
(0.764)\end{array}$ & $\begin{array}{l}-0.320 \\
(0.391)\end{array}$ & -3.18 & -2.16 & -1.57 \\
\hline GP6 & & & & & & \\
\hline GP7 & $\begin{array}{l}0.496^{1 /} \\
(0.157)\end{array}$ & $\begin{array}{l}0.442^{2 /} \\
(0.187)\end{array}$ & $\begin{array}{l}1.193^{1 /} \\
(0.145)\end{array}$ & 4.00 & 1.45 & 5.87 \\
\hline Constant & $\begin{array}{l}1.539^{1 /} \\
(0.109)\end{array}$ & $\begin{array}{l}0.659^{1 /} \\
(0.145)\end{array}$ & $\begin{array}{l}1.773^{1 /} \\
(0.122)\end{array}$ & & & \\
\hline Observations & 41 & 41 & 31 & & & \\
\hline LR Chi-squared & 104.6 & 68.23 & 39.07 & & & \\
\hline prob>chi2 & 0.00 & 0.00 & 0.00 & & & \\
\hline Pseudo R2 & 0.42 & 0.38 & 0.36 & & & \\
\hline
\end{tabular}

Fuente: elaboración propia. 


\subsection{Significancia estadística de los estimadores}

Los estimadores resultantes de la regresión Poisson son los que se muestran en las tres primeras columnas de la Tabla 3. En ninguna de las tres matrices de los EIA utilizados, existían actividades pertenecientes al Grupo GP6; es decir, aquellas que afectan solo al medio biológico (aparece la fila vacía).

La significancia conjunta de los estimadores, en los tres sub-proyectos evaluados, son 104.6, 68.2 y 39.1 (valores Chi-Squared), asociados a probabilidades $<0.05$ en los tres casos. O sea, en los tres modelos, en forma global, los resultados son robustos y consistentes por el estadístico de prueba "razón de verosimilitud" (estadístico Ji-cuadrado ${ }^{25}$ ). La bondad de ajuste, apreciada a través del esta-

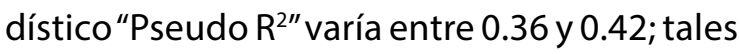
indicadores son usualmente bajos en todos los modelos probabilísticos y son considerados de segunda importancia en la indicación de la pertinencia de un modelo; podría concluirse sin embargo que, en promedio, 38\% de la variabilidad de la variable dependiente es explicada por los regresores considerados (Wooldridge, 2009, pp. 628).

En forma individual, la mayoría de estimadores de la relación de variables exógenas $\left(\beta_{j}\right)$ son significativos en los tres modelos, pues sus valores $p$-value son menores al $5 \%$. La excepción (en los tres casos) la constituye la

25 Con el método de máxima verosimilitud el estadístico Jicuadrado sirve para probar la hipótesis conjunta de significancia estadística de todos los estimadores de los parámetros de los modelos multivariados (es el equivalente al estadístico "F" en el modelo clásico estimado mediante Mínimos Cuadrados Ordinarios). variable exógena que representan al grupo de actividades GP5 (a las que les corresponde $p$-value de $0.58,0.43$ y 0.3 , en los tres modelos, respectivamente); ello se debería a que las actividades de este grupo GP5, habrían sido más impactantes de lo informado en los EIA (por tanto "sesgadas" en favor del operador).Es interesante notar, que esto sucede en los tres sub-proyectos; es decir que en los tres casos las actividades que afectan solo al medio físico tendrían problemas de evaluación, por lo que se sugiere tener especial cautela, por parte de las instituciones, en el momento de los monitoreos, una vez implementado el sub-proyecto o sub-proyectos evaluados.

Adicionalmente, otro grupo de actividades que también ha mostrado evidencia de dificultades en cuanto a la evaluación de impacto efectuada en el EIA, son las que constituyen el grupo que afectan al medio físico y biológico, en el caso del sub-proyecto de la "Planta de Gas" (esto es evidenciado también por la falta de significancia del estimador del parámetro que mide el efecto del grupo GP2, según Tabla 3); sin embargo, esto no ocurre con el estimador de la matriz BID.

\subsection{Efectos Marginales}

Siendo necesario medir los efectos marginales, para ser exhaustivos en la validación, en las dos últimas columnas de la Tabla 3, se muestra el cálculo del efecto marginal o parcial de cada grupo analizado en el modelo respectivo. Tal efecto marginal o parcial, representa el efecto de un incremento de una unidad en el valor de GPi sobre la media (promedio) de FACT como variable dependiente. 
Es decir utilizando la ecuación (7):

$$
\frac{\partial \lambda_{i}}{\partial X_{j i}}=\beta_{k} \lambda_{i}
$$

Es importante destacar que las tasas de cambio del valor promedio de los impactos, con respecto al efecto de las actividades agrupadas en los diferentes grupos $\mathbf{G P i}$ son casi todas negativas (excepto el caso de las actividades del grupo GP7). Tales valores negativos varían entre -7.13 en el caso de GP1 en el Sub Proyecto "planta de gas" y -0.49 en el caso de GP2 también en el Sub Proyecto "planta de gas".

El valor de -7.13 , por ejemplo, quiere decir, que ante un cambio en una unidad en el cualquiera de las actividades que pertenecen a GP1, permaneciendo constantes los demás grupos de actividades, la esperanza de cambio (o cambio marginal promedio) en el conjunto de factores ambientales y sociales es de -7.13 veces; es decir, se produce un efecto negativo en el $\mathrm{N}^{\circ}$ promedio de veces que los factores (ambientales y sociales) son afectados. Así mismo, si se incrementara el coeficiente de las actividades de GP2 (actividades que afectan al medio físico y biológico en este caso) en una unidad, se produciría también un efecto negativo en el número promedio de veces que los factores ambientales y sociales son afectados. (Tabla 3).

Los valores positivos de los efectos marginales mostrados en la Tabla 3, para el caso de las actividades de GP7 (que afectan solo al medio social), indican lo siguiente: en el caso del valor 5.87 (por ejemplo), un incremento en una unidad en el conjunto de acciones que per- tenecen a GP7, permaneciendo constante los demás grupos de actividades, la esperanza de cambio en el $\mathrm{N}^{\circ}$ de impactos promedio (en el conjunto de factores ambientales y sociales) incrementaría en casi 6 veces. En lo que corresponde a la tasa de cambio que reportó un valor de 1.45 (también actividades del grupo GP7), se interpreta que ante un cambio en una unidad en el conjunto de acciones que pertenecen a GP7, permaneciendo constante los demás grupos de actividades, la esperanza de cambio por $\mathrm{N}^{\circ}$ de impactos en el conjunto de factores ambientales y sociales es de casi 1 vez y media más (1.45).

\section{CONCLUSIONES}

a. Se ha comprobado la aplicabilidad y funcionalidad de la metodología para validar las matrices de impacto, como la de los subproyectos de CAMISEA, mediante el modelo Poisson que usa "datos de cuenta". Es posible generalizar el método para otro conjunto de valoraciones cualitativas distintas, basadas en el uso de las Matrices Leopold o semejantes, que en su constructo incorporan múltiples criterios para su evaluación cualitativa y que serán de gran utilidad a las autoridades competentes para tomar decisiones sobre la viabilidad de los proyectos energéticos y mineros, ya que estos serían sostenibles en el tiempo.

b. La significancia individual de los estimadores del modelo econométrico usado es fundamental para validar la pertinencia y equidad de las evaluaciones de 
los EIA, basadas cualitativamente en la construcción de las Matrices de Leopold. Mediante tal significancia individual, es posible identificar las actividades que habrían sido regular o mal evaluadas en su impacto (por tanto sesgadas en favor del operador) y que requerirían una mayor observación durante la fase de aplicabilidad, para evitar mayores impacto en el mediano y largo plazo del proyecto, a fin de cautelar una sostenibilidad intertemporal del proyecto en evaluación.

c. El método puede ser fácilmente aplicable tanto a casos de matrices construidas en base a métodos estandarizados y formales de evaluación de impacto (como las metodologías de CONESA y BID), así como en los casos menos formales aplicados por los equipos multidisciplinarios contratados por las Empresas Operadoras de los proyectos). En el caso de CAMISEA, resultó interesante observar que el grupo de actividades GP5, es decir actividades que afectan al medio físico, resultó con indicadores que recurrentemente evidenciaron dificultades potenciales en los tres sub-proyectos utilizados.

d. Los efectos marginales expresan los cambios en los valores promedio de los impactos totales en los medios físicos, biológicos y sociales, como consecuencia de cambios en las variables independientes (que representan el conjunto de actividades que afectan los factores). Un valor positivo, expresa una relación directa entre cambios en las actividades impactantes y los factores impactados $\left(\mathrm{N}^{\circ}\right.$ de impactos); en cambio estimadores negativos expresan una relación inversa entre los cambios en las actividades regresoras y el valor promedio de los factores impactados, observándose el potencial impacto que generan las externalidades. En el caso de los dos sub-proyectos de CAMISEA (lote 88) tales efectos marginales resultaron mayoritariamente negativos.

e. Este estudio tiene básicamente un carácter demostrativo de la funcionalidad del modelo y del método de evaluación de matrices de impacto, es por ello que se han usado solo tres EIA, para tal propósito. En tal sentido no se ha creído conveniente hacer recomendaciones a partir de un grupo relativamente pequeño de actividades, en el caso CAMISEA. Así mismo, en este caso se han agrupado actividades en grupos más o menos homogéneos pero en forma algo subjetiva; podría realizarse tal agrupación en diferente forma y de modo tal que fuera posible tener estimadores mucho más (o menos) detallados que en presente caso. 


\section{REFERENCIAS BIBLIOGRÁFICAS}

ÁLVAREZ, B. (2001), "La demanda atendida de consultas médicas y servicios urgentes en España", en Investigaciones Económicas, XXV (1): 93-138.

AZQUETA OYARZUN, DIEGO (1994),Valoración Económica de la Calidad Ambiental, McGraw-Hill Editores.

BANCO INTERAMERICANO DE DESARROLLO (BID, 2001),Centro de Estudios para el Desarrollo (CED), Autor: Guillermo Espinoza, Santiago de Chile, Chile.

CAMERON, A.C., TRIVEDI, P.K., MILNE, F. Y J. PIGGOTT (1988), "A microeconometric model of the demand for health care and health insurance in Australia". Review of Economic Studies, LV: 85-106.

CAMERON, A.C. Y TRIVEDI, P.K., (2013), Regression Analysis of Count Data, printed by The Econometric Society Monographs, the United State of America, Second Edition.

CONESA V., VÍTORA (2010), Guía Metodológica para la Evaluación del Impacto Ambiental. 4ta edición, Editorial Mundi Prensa.

GUJARATI, DAMODAR N. (2009), Econometría, Editorial Mc Graw-Hill. Quinta Edición.

GREENE, WILLIAM (2004), Análisis Econométrico,Quinta Edición. Editorial Prentice Hall, Madrid- España.

ENVIRONMENTAL RESOURCES MANAGEMENT (ERM, 2011), Estudio de Impacto Ambiental y Social del Lote 88, Camisea y Área de influencia, Consultora Internacional ERM, Agosto de 2001.
GERDTHAM, U.G. (1997), “Equity in health care utilization: further tests based on hurdle models and Swedish micro data", in Health Economics6 (1): 303-319.

MUNDA, GIUSEPPE(2003), "Social multi-criteria evaluation: Methodological foundations and operational consequences", in European Journal of Operational Research, 158 (2004) 662-677.

MUNDA, GIUSEPPE (2008), Social multi-criteria evaluation for a sustainable economy. Springer-Verlag Berlin Heidelberg. 210 pag.

WOOLDRIDGE, JEFFREY (2009), Introducción a la Econometría: un Enfoque Moderno, CENGAGE Learning Editores.

PALOMARES, CARLOS (TESIS no publicada),"Medición de Impactos en los Recursos Naturales Comunes del Proyecto Camisea-Perú, a través de la Evaluación de Estudios Individuales de Impacto Ambiental, Utilizando Datos Panel" (borrador final de TESIS).

POHLMEIER, W. and ULRICH, V. (1995), "An Econometric Model of the Two-Part Decisionmaking Process in the Demand for Health Care", in Journal of Human Resources, 30(2): 339-361.

RIERA, P., GARCÍA, D., KRISTROM, B. y BRANNLUND, R. (2005), Manual de Economía Ambiental y de los Recursos Naturales. Editorial Thomson, España (350 pp.)

URBANOS, R. (2000), “Desigualdades socio sanitarias y efectividad potencial de las políticas públicas: un estudio aplicado con datos españoles", en Hacienda Pública Española 154 (3): 217-238. 


\section{ANEXOS}

Anexo 1. Matriz de Impacto Ambiental N²0: Lote 88 - Sub Proyecto Planta de Gas y Área de Servicios - Fase Construcción

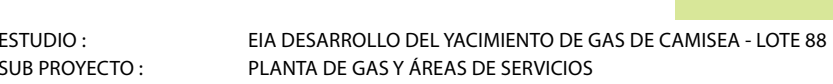

FASE CONSTRUCCIOON

$\begin{array}{ll}\text { OPERADOR: } & \text { PLUSPETROL PERÚ CORPORATION S.A. } \\ \text { CONSULTORA: } & \text { ERM PERU S.A. }\end{array}$

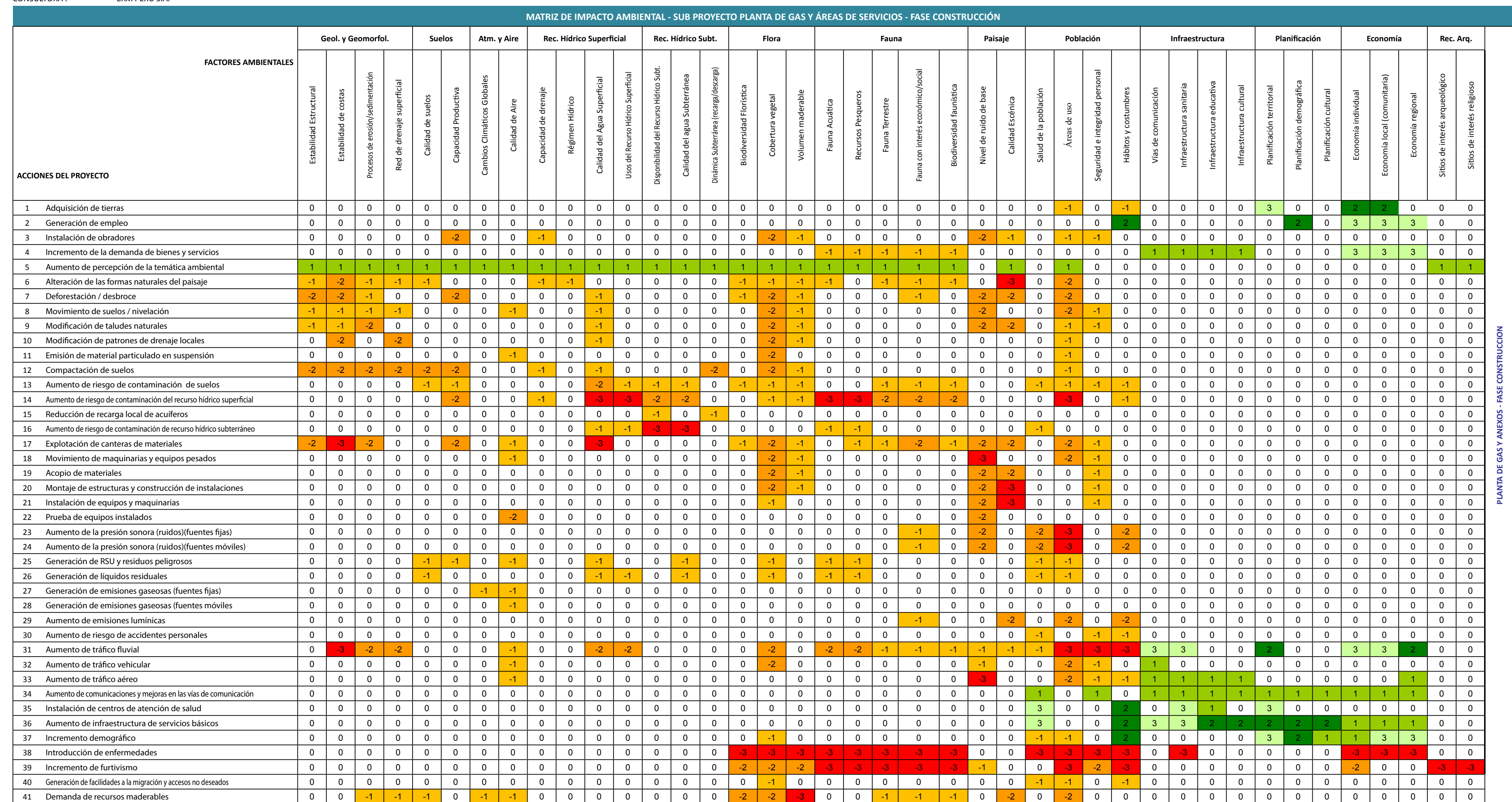

\begin{tabular}{|ccccc|}
\hline \multirow{3}{*}{ IMPACTOS NEGATIVO } & 1 & $\begin{array}{c}\text { INTENSIDAD } \\
\text { LEVE }\end{array}$ & 1 & \\
& 2 & MODRADO & 2 & IMPACTOS POSITIVOS \\
& 3 & SIGNIFICATIVO & 3 & \\
\hline
\end{tabular}


Anexo 2. DO FILE DE VALIDACION DE MATRICES

TESIS DOCTORAL NO PUBLICADA (SETIEMBRE-2013)

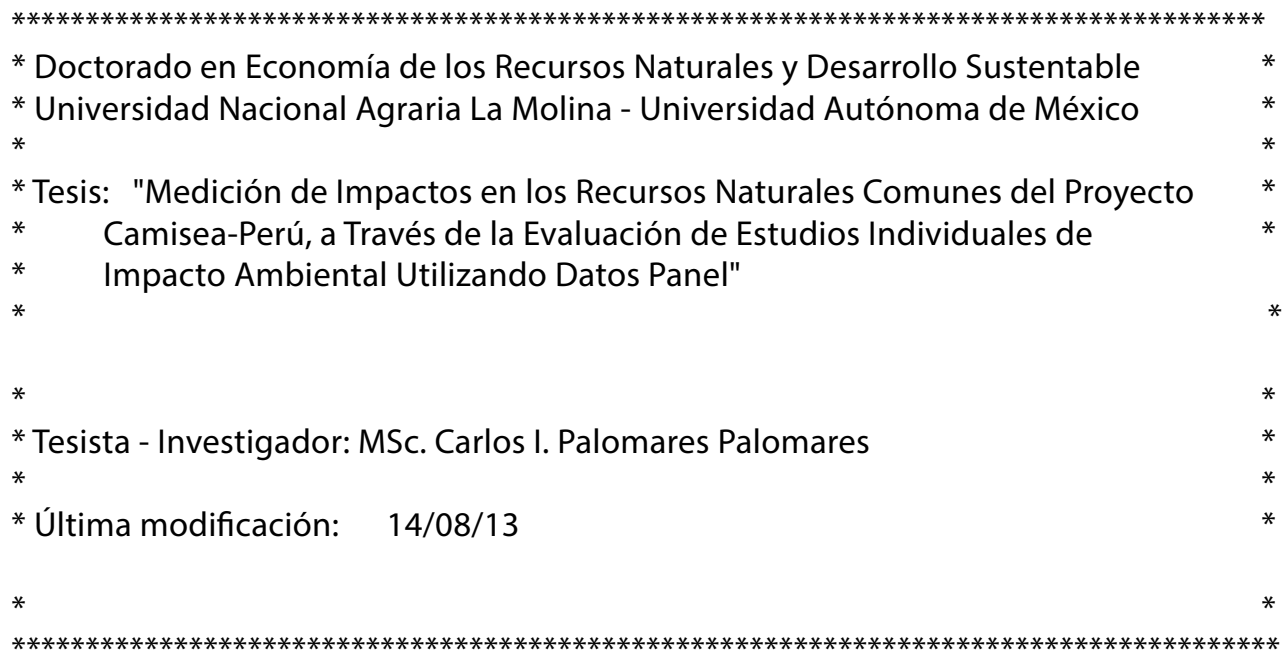

set more off

cd "C: \Users \Toshiba\Desktop \Data_cipp(vmatriz)"

use "data_vmatriz", clear

capture log close

log using "data_vmatriz", replace

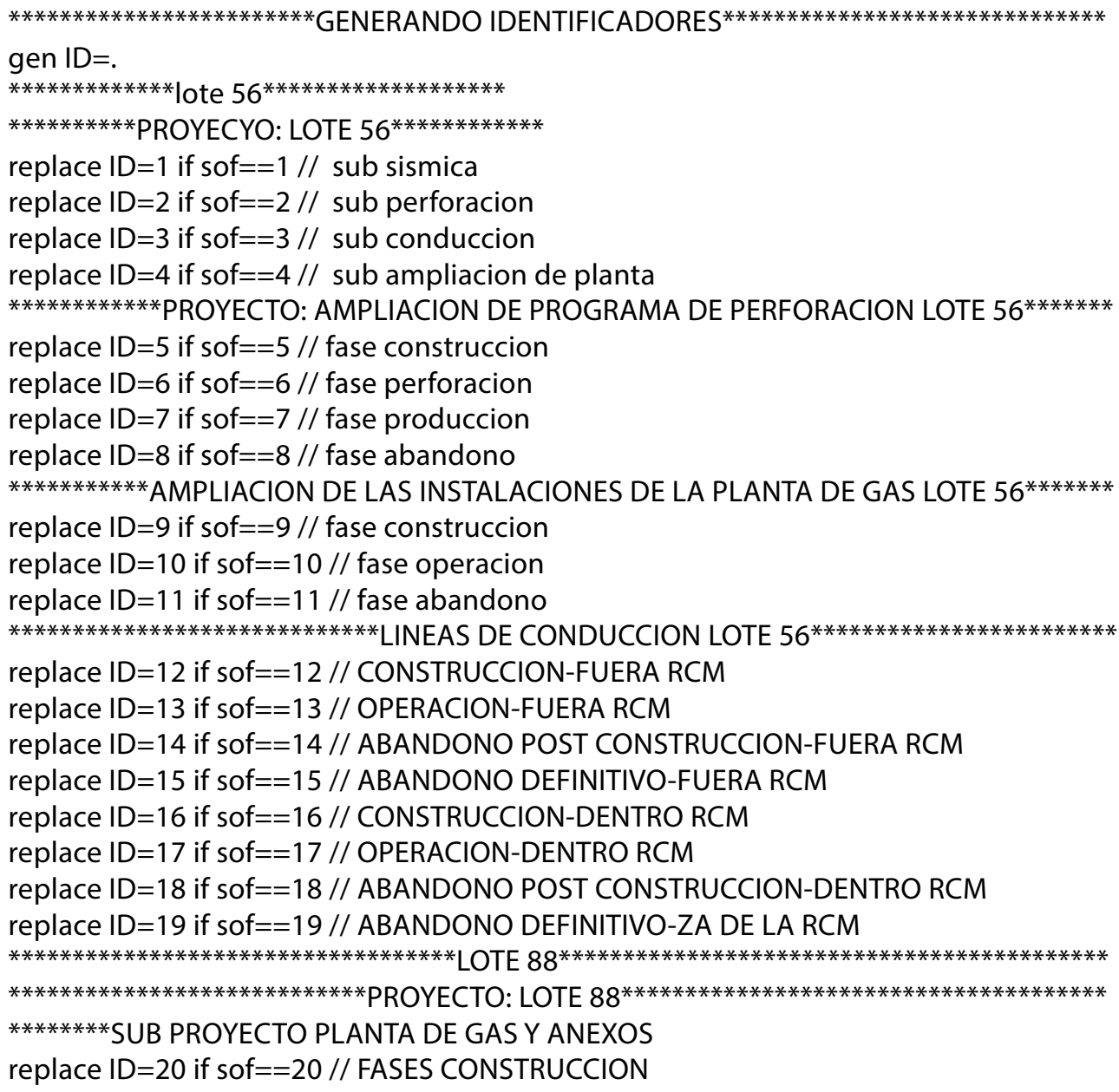


replace ID $=21$ if sof $==21 / /$ FASE OPERACION replace ID $=22$ if sof $==22 / /$ FASE ABANDONO *******SUB PROYECTO LINEAS DE CONDUCCION replace ID $=23$ if sof $==23 / /$ FASE CONSTRUCCION replace ID $=24$ if sof $==24 / /$ FASE OPERACION replace ID $=25$ if sof $==25 / /$ FASE ABANDONO *********SUB PROYECTO PERFORACION replace ID $=26$ if sof $==26 / /$ FASES PERFORACION Y TESTEO replace ID $=27$ if sof $==27 / /$ FASES ABANDONADO $* * * * * * *$ SUB PROYECTO SISMICA 3D replace ID $=28$ if sof $==28$ // FASES OPERACIONES PREVIAS Y ADQUISICION DE DATOS replace $I D=29$ if sof $==29 / /$ FASES ABANDONO

$* * * * * * * * * * * * * * * * * * * * * * * * * * * * * * * * * * * * * * * * * * * * * * * * * * * * * * * * * * * * * * * * * * * * * * * * * * * * * * *$

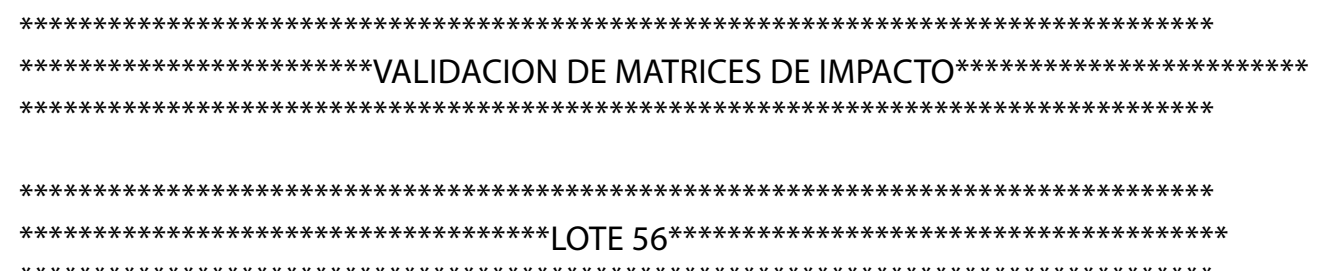

$* * * * * * * * * * * * * * * * * * * * * * * * * * * * * * * * * * * * * * * * * * * * * * * * * * * * * * * * * * * * * * * * * * * * * * * * * * * * * * * * *$

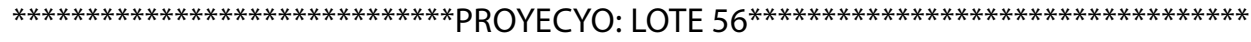

**SUB PROYECTO SISMICA

poisson fact gp1 gp3 gp4 gp7 if ID==1

**SUB PROYECTO PERFORACION

poisson fact gp1 gp3 gp4 if $I D==2$

* SUB PROYECTO CONDUCCION

poisson fact gp1 gp3 if ID==3

**SUB PROYECTO AMPLIACION DE PLANTA

poisson fact gp1 gp2 gp3 gp6 gp7 if ID==4

******************PROYECTO: AMPLIACION DE PROGRAMA DE PERFORACION LOTE $56^{* * * * * * *}$

**FASE CONTRUCCION

poisson fact gp1 gp2 gp3 gp4 gp5 gp7 if ID==5

**FASE PERFORACION

poisson fact gp1 gp2 gp3 gp4 gp5 gp7 if ID==6

**FASE PRODUCCION

poisson fact gp2 gp3 gp5 gp7 if ID==7

**FASE ABANDONO

poisson fact gp2 $\mathrm{gp} 5$ if $I D==8$

**************AMPLIACION DE LAS INSTALACIONES DE LA PLANTA DE GAS LOTE $56^{* * * * * * *}$

**FASE CONTRUCCION

poisson fact gp1 gp2 gp4 gp5 pg6 gp7 if ID==9

**FASE OPERACION

poisson fact gp1 gp2 gp5 pg6 if ID==10

**FASE ABANDONO

poisson fact gp2 gp5 if $I D==11$

**************LINEAS DE CONDUCCION LOTE $56^{* * * * * * * * * * * * * * * *}$

**CONSTRUCCION-FUERA RCM

poisson fact gp1 gp2 gp3 gp4 gp7 if ID==12

**OPERACION-FUERA RCM

poisson fact gp1 gp2 gp3 gp5 gp7 if ID==13

**ABANDONO POST CONSTRUCCION-FUERA RCM

poisson fact gp1 gp2 gp3 gp4 gp7 if ID==14

${ }^{* *}$ ABANDONO DEFINITIVO-FUERA RCM 
poisson fact gp1 gp2 gp3 gp4 gp7 if ID==15

**CONSTRUCCION-DENTRO RCM

poisson fact gp1 gp2 gp3 gp4 gp7 if ID==16

**OPERACION-DENTRO RCM

poisson fact gp1 gp2 gp3 gp5 gp7 if $\mathrm{ID}==17$

**ABANDONO POST CONSTRUCCION-DENTRO RCM

poisson fact gp1 gp2 gp3 gp4 gp7 if $\mathrm{ID}==18$

**ABANDONO DEFINITIVO-ZA DE LA RCM

poisson fact gp1 gp2 gp3 gp4 gp7 if ID==19

************************************************************************************1

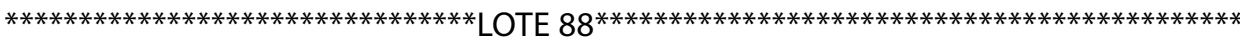

*****************************************************************************

$* * * * * * * * * * * * * * * * * * * * * * * * * *$ PROY CCTO: LOTE $88 * * * * * * * * * * * * * * * * * * * * * * * * * * * * * * * * * * *$

********SUB PROYECTO PLANTA DE GAS Y ANEXOS

********ASES

*****CONSTRUCCION

poisson fact gp1 gp2 gp3 gp5 gp7 if ID==20

*****OPERACION

poisson fact gp1 gp2 gp3 gp7 if ID==21

*****ABANDONO

poisson fact gp1 gp2 gp3 gp4 gp5 gp7 if ID==22

********SUB PROYECTO LINEAS DE CONDUCCION

********FASES

*****CONSTRUCCION

poisson fact gp1 gp3 gp4 gp7 if ID==23

*****OPERACION

poisson fact gp1 gp3 gp4 gp5 gp7 if ID==24

*****ABANDONO

poisson fact gp1 gp3 gp4 gp6 gp7 if ID==25

********SUB PROYECTO PERFORACION

*******FASES

*****PERFORACION Y TESTEO

poisson fact gp1 gp2 gp3 gp4 gp5 gp7 if $\mathrm{ID}==26$

*****ABANDONADO

poisson fact gp1 gp2 gp3 gp4 gp5 gp7 if ID==27

********SUB PROYECTO SISMICA 3D

*******FASES

*****OPERACIONES PREVIAS Y ADQUISICION DE DATOS

poisson fact gp1 gp3 gp4 gp7 if ID==28

${ }^{* * * * *}$ ABANDONO

poisson fact gp1 gp3 gp5 gp6 if ID==29

log close 